\title{
Improved High Dynamic Range for 3D Shape Measurement based on Saturation Of the Coloured Fringe
}

\author{
Shanyu Chua ${ }^{1 *}$, Chee Chin Lim ${ }^{1}$, Swee Kheng Eng ${ }^{1}$, Yen Fook Chong ${ }^{1}$ and \\ Chiun Tai Loh ${ }^{2}$ \\ ${ }^{1}$ School of Mechatronic Engineering, Universiti Malaysia Perlis, Pauh Putra Campus, \\ 02600 Arau, Perlis, Malaysia \\ ${ }^{2}$ Research and Developemt Department, Estek Automation Sdn Bhd, Bayan Lepas, \\ 11900 Pulau Penang, Malaysia
}

\begin{abstract}
Phase-shifting fringe projection methods have been developed for three-dimensional scanning (Zuo et al., 2018). However, the 3-Dimensional (3D) scanning of objects with a high dynamic reflectivity range based on structured light is a challenging task to achieve (Feng et al., 2018). The incorrect intensities captured will cause phase and measurement errors. Thus, this paper proposes a method that improves the current High Dynamic Range (HDR) (Jiang et al., 2016)) method to increase the dynamic range. The camera and projector have 3 channels, red, green, and blue, which can absorb and project these lights independently. This paper proposes a method that makes use of this by controlling the intensity of each projected for the camera. Each image can be split into 3 channels and provide 3 images which contain different intensities, then it will be used to compute the $3 \mathrm{D}$ information. In general, this is done by controlling the projection of red, green and blue (RGB) channel and apply the Jiang's algorithm (Jiang et al., 2016). The results are compared and analysed with current HDR (Jiang's method) and the regular three-step phase-shifting methods. From the experimental results, it has shown that our proposed method outperforms the current HDR and the regular three-step

ARTICLE INFO

Article history:

Received: 18 October 2020

Accepted: 23 February 2021

Published: 30 April 2021

DOI: https://doi.org/10.47836/pjst.29.2.04

$\overline{\text { E-mail addresses: }}$

phase-shifting methods. Specifically, the proposed method manages to increase the dynamic range of the reflective property of objects. Additionally, our proposed method has also significantly reduced the times of 3D object measurements.
\end{abstract}

shanyuchua.ed@gmail.com (Shanyu Chua)

cclim@unimap.edu.my (Chee Chin Lim)

kheng@unimap.edu.my (Swee Kheng Eng)

yenfook@unimap.edu.my (Yen Fook Chong)

$*$ Corresponding author
Keywords: 3D scanning, fringe profilometry, phaseshifting profilometry 


\section{INTRODUCTION}

Machine vision is a popular technology used in industry for automatic inspection and analysis. This method is widely applied in various fields, such as automatic inspection in manufacturing, process control, robot guidance, medical field and entertainment. The main reason for the wide application of this technology in various industries is due to its non-contact property that allows inspection or measurement on material that are soft or easily deformable objects which are usually not suitable for contact inspection (Zuo et al., 2018). It improves the productivity and the quality of the product and provides a competitive advantage to industries (Malamas et al., 2003). The rapidly evolving of computers and image capturing device has allowed this technology to be used in real-time optical inspection system with high accuracy (Huang \& Pan, 2015).

Currently, due to the rapid evolution of technology, non-contact 3D shape measurement can acquire fast and precise data. There are many approaches for the 3D shape measurement methods, such as optical interferometry (Pavlicek \& Mikeska, 2018), time-of-flight technique (Achar et al., 2017), stereo vision (Zhang, 2018), laser triangulation (Faes et al., 2016), and structured light (Aboali et al., 2017). One of the popular methods is the structured light method and it has been extensively researched and applied in the industries due to its easy hardware setup, high accuracy of measurement, high-density level, high speed and inexpensive (Zhang \& Huang, 2006b; Zuo et al., 2018). Fringe projection profilometry (FPP) is the method that projects light with known patterns and applies the triangulation method to obtain the 3D information of the object (Liu et al., 2010).

However, measuring the 3D information of an object's surface with a wide variety of reflective properties and contrast can prove to be a difficult task to perform, especially to achieve consistent high-quality measurement across the entire object (Jiang et al., 2016). For the darker surfaces, lower exposure or low light intensity is used, and higher exposure or high light intensity is used for the brighter surfaces. The specular reflection which is caused by the object with a shiny surface will be acquired as white pixels because of the high reflection of light (Feng et al., 2018). This problem can be solved by spraying a thin layer of diffused powder but this method is not suitable for all objects and some measurements require high precision where the thin layer of spray cannot be ignored (Feng et al., 2018). Therefore, there is a great need for HDR techniques which can overcome this shortcoming.

HDR techniques refer to methods that are used to calculate the surface with a wide variety of surface reflectivity properties (Jiang et al., 2016). Currently, a few HDR techniques have been studied and developed to overcome this problem, such as applying a polarizing filter (Salahieh et al., 2014; Wolff, 1989), capturing images at multiple exposures (Babaie et al., 2015; Song et al., 2017; Zhang \& Yau, 2009), capturing images at different light projection intensities (Waddington \& Kofman, 2014), and applying algorithm (Chen et al., 2018; Jiang et al., 2016; Wang et al., 2017). The method proposed in Babaie et al. 
(2015), Song et al. (2017), Waddington \& Kofman (2014), and Zhang \& Yau (2009) requires many images which are time-consuming. In the year 2016, Jiang et al. (2016) developed an algorithm that did not rely on the change of camera exposure or the intensity of the projector. By using the three-step phase-shifting fringe (3PSP) and inverse three-step phase fringe, the inverted fringe can be used to complement the saturated pixels. However, it is unable to reconstruct object with surface reflectivity that has a higher dynamic range.

Inspired by Jiang et al. (2016), this paper proposes to enhance the HDR method by projecting controlled red, green and blue (RGB) channel intensity to increase the dynamic range of surface measurement $t$ is done by projecting the fringe at different RGB channel intensity. The fringe is then split to their respective channel and is used for the phase calculation. Therefore, by controlling the RGB intensity that project brightness that is suitable for the surface reflectivity, Jiang's HDR method can be enhanced.

\section{METHOD}

\section{Three-Step Phase-Shifting}

The fringe is first generated using the computer. In this experiment, 3PSP is used. 3PSP needs 3 images of fringe pattern with phase-shifts at $-120^{\circ}, 0^{\circ}$ and $120^{\circ}$ to obtain the $3-\mathrm{D}$ data (Zhang, 2016; Zhang \& Huang, 2006a). The fringe pattern is generated using Equation 1, 2, and 3:

$$
\begin{aligned}
& I_{1}(x, y)=I^{\prime}(x, y)+I^{\prime \prime}(x, y) \cos [\varnothing(x, y)-\alpha] \\
& I_{2}(x, y)=I^{\prime}(x, y)+I^{\prime \prime}(x, y) \cos [\varnothing(x, y)] \\
& I_{3}(x, y)=I^{\prime}(x, y)+I^{\prime \prime}(x, y) \cos [\varnothing(x, y)+\alpha]
\end{aligned}
$$

where, $I_{1}, I_{2}$, and $I_{3}$ are images with phase-shift at $-120^{\circ}, 0^{\circ}$, and $120^{\circ}, I^{\prime}$ is the mean intensity, $I^{\prime \prime}$ is the peak-to-valley intensity modulation, $\varnothing(x, y)$ is the wave front phase, and $\alpha$ is phase shift at $120^{\circ}$. The phase, $\varnothing(x, y)$ as shown in Equation 4 , can be obtained by using Equation 1, 2, and 3.

$$
\emptyset(x, y)=\tan ^{-1} \frac{\sqrt{3}\left[I_{1}(x, y)-I_{3}(x, y)\right]}{2 I_{2}(x, y)-I_{1}(x, y)-I_{3}(x, y)}
$$

This method is not suitable for scanning objects with a high surface reflectivity because the dark region of the sample captured will be under-exposed while the highly reflective surface is over-exposed. Hence, this cause difficulty in capturing the desired result when 
both kinds of surfaces are present (Feng et al., 2018). To overcome this problem, Jiang proposed a HDR method (Jiang et al., 2016) that used the inverted 3PSP to complement the regular 3PSP shown in Current HDR Method.

\section{Current HDR Method}

Another 3 inverse fringes are generated, which serve to increase the contrast of the 3D surface to be measured, using Equation 5, 6, and 7.

$$
\begin{aligned}
& I_{1}^{i n v}(x, y)=I^{\prime}(x, y)-I^{\prime \prime}(x, y) \cos [\varnothing(x, y)-\alpha] \\
& I_{2}^{i n v}(x, y)=I^{\prime}(x, y)-I^{\prime \prime}(x, y) \cos [\varnothing(x, y)] \\
& I_{3}^{i n v}(x, y)=I^{\prime}(x, y)-I^{\prime \prime}(x, y) \cos [\varnothing(x, y)+\alpha]
\end{aligned}
$$

Two sets of fringes are generated, where one of it contain fringe with 6-pixel width and 120-pixel width fringe (Zhang, 2016; Zhang \& Huang, 2006a). Each set of fringes contain 6 images.

The phase will be computed using Jiang's method (Jiang et al., 2016). When $I_{1}(x, y)$ is saturated, it is replaced with $I_{1}^{i n v}(x, y)$ and using Equation 8. When $I_{2}(x, y)$ is saturated, it is replaced with $I_{2}^{i n v}(x, y)$ and using Equation 9. When $I_{3}(x, y)$ is saturated, it is replaced with $I_{3}^{i n v}(x, y)$ and using Equation 10 . When $I_{1}(x, y)$ and $I_{2}(x, y)$ are saturated, it is replaced with $I_{1}^{i n v}(x, y)$ and $I_{2}^{i n v}(x, y)$, and using Equation 11. When $I_{1}(x, y)$ and $I_{3}(x, y)$ are saturated, it is replaced with $I_{1}^{i n v}(x, y)$ and $I_{3}^{i n v}(x, y)$ and using Equation 12. When $I_{2}(x, y)$ and $I_{3}(x, y)$ are saturated, it is replaced with $I_{2}^{i n v}(x, y)$ and $I_{3}^{i n v}(x, y)$, and using Equation 13. If $I_{1}(x, y), I_{2}(x, y)$ and $I_{3}(x, y)$, then Equation 14.

$$
\begin{aligned}
& \emptyset(x, y)=\tan ^{-1} \frac{-3 I_{1}^{i n v}(x, y)+2 I_{2}(x, y)+I_{3}(x, y)}{\sqrt{3}\left[I_{1}^{i n v}(x, y)-I_{3}(x, y)\right]} \\
& \varnothing(x, y)=\tan ^{-1} \frac{I_{1}(x, y)-I_{3}(x, y)}{\sqrt{3}\left[I_{1}(x, y)+I_{3}(x, y)-2 I_{2}^{i n v}(x, y)\right]} \\
& \varnothing(x, y)=\tan ^{-1} \frac{-I_{1}(x, y)-2 I_{2}(x, y)+3 I_{3}^{i n v}(x, y)}{\sqrt{3}\left[I_{3}^{i n v}(x, y)-I_{1}(x, y)\right]}
\end{aligned}
$$




$$
\begin{aligned}
& \emptyset(x, y)=\tan ^{-1} \frac{I_{1}^{i n v}(x, y)+I_{2}^{i n v}(x, y)-3 I_{3}(x, y)}{\sqrt{3}\left[I_{1}^{i n v}(x, y)-I_{3}(x, y)\right]} \\
& \varnothing(x, y)=\tan ^{-1} \frac{I_{3}^{i n v}(x, y)-I_{1}^{i n v}(x, y)}{\sqrt{3}\left[2 I_{2}(x, y)-I_{1}^{i n v}(x, y)-I_{3}^{i n v}(x, y)\right]} \\
& \varnothing(x, y)=\tan ^{-1} \frac{3 I_{1}(x, y)-I_{2}^{i n v}(x, y)-I_{3}^{i n v}(x, y)}{\sqrt{3}\left[I_{3}^{i n v}(x, y)-I_{1}(x, y)\right]} \\
& \varnothing(x, y)=\tan ^{-1} \frac{2\left[B_{1}(x, y)-B_{3}(x, y)\right]}{\sqrt{3}\left[B_{2}(x, y)-B_{1}(x, y)-B_{3}(x, y)\right]}
\end{aligned}
$$

wh e r e $B_{1}(x, y)=I_{1}(x, y)-I_{1}^{i n v}(x, y), \quad B_{2}(x, y)=I_{2}(x, y)-I_{2}^{\text {inv }}(x, y), \quad$ a n d $B_{3}(x, y)=I_{3}(x, y)-I_{3}^{i n v}(x, y)$.

\section{Enhanced HDR Method}

Even though the current method is faster and does not require multiple exposures of images, however, it tends to fail during measurement of wide ranges of reflectivity due to only a single exposure (Feng et al., 2018). Thus, our enhanced HDR method is proposed.

With the aim of increasing the dynamic range of the surface from the current HDR method, the image is captured by controlling the RGB intensity. The projector will project the red channel that is set to the lowest intensity, the blue channel that is set to the highest intensity and green channel that is set to the mid-intensity. With this, 3 intensities can be obtained from a single set of images. Each set of images from these 3 images can be used independently to compute the $3 \mathrm{D}$ coordinates.

The overall flow chart for this method is as shown in Figure 1. It will start from the channel with the highest intensity, which is the blue channel, and check if all three non-inverse fringes are over-exposed. If it is not over-exposed, it will be used for 3D coordinates computation; and if it is over-exposed, it will proceed to check the second highest intensity channel which is the green channel. Green channel will be checked if all three non-inverse fringes are over-exposed. If it is not over-exposed, it will be used for 3D coordinates computation; and if it is over-exposed, it will proceed to the lowest intensity channel, which is the red channel. If all three non-inverse fringes are still over-exposed, computation will be done using Equation 14. 


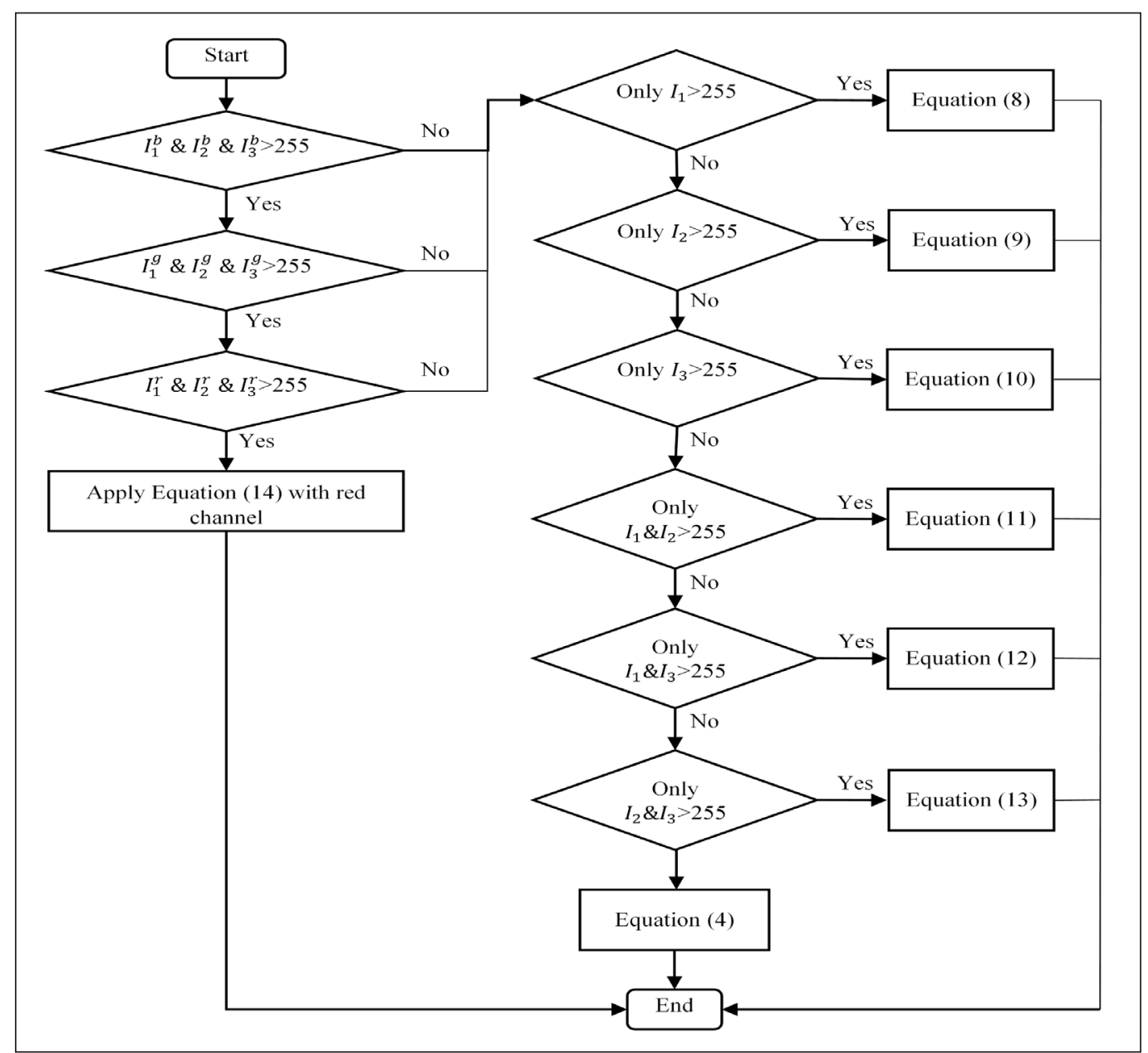

Figure 1. The flowchart of the proposed method

\section{Experiment Setup}

The camera used was Basler axA2040-55uc USB 3.0 with Sony IMX265 Complementary Metal-Oxide-Semiconductor (CMOS) sensor. The lens used was TCSM024: 3D bitelecentric lens with Scheimpflug adjustment. The projector used was a digital light projection (DLP). LightCrafter 4500 was a software used to flash the projector's memory and control the projector. pylonViewer was used to view and capture the image from the camera. The captured image was then processed using the proposed method. Figure 2 demonstrates the setup of the experiment.

\section{RESULTS AND DISCUSSION}

The experiment was done using a sample with high dynamic reflective surfaces. The sample used was a circuit board as shown in Figure 3(a). From Figure 3(a), there are four main 


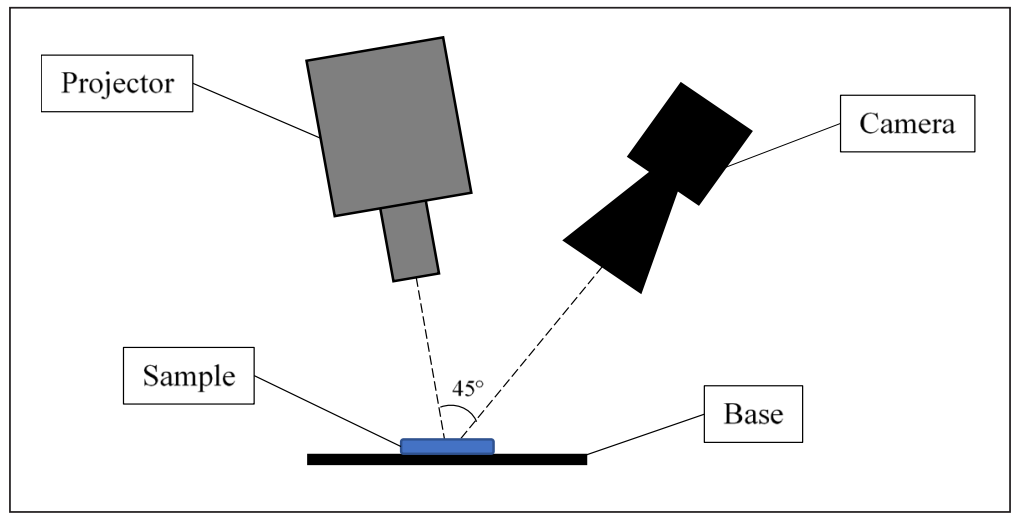

Figure 2. Diagram of the experimental setup

classes of components, as labelled using 1,2,3 and 4. Component class 1 and component class 2 consist of a middle Integrated Circuit (IC) and the biggest Integrated Circuit (IC), respectively. Component class 3 consists of three smallest Integrated Circuits (ICs), and the component class 4 consists of ten resistors. For all the Integrated Circuits (ICs), the low reflective property is at the semiconductor wafers (black colour) part, and the high reflective property is at the soldered part. Figure 3(d) shows the current HDR method's result which is better compared to Figure 3(b), and Figure 3(c). These experimental results show that the proposed HDR method has a great improvement in the accuracy to measure objects with high-contrast surfaces.

Figure 4 shows the result of the experiment using a different light setting and luminance. The experiment done by capturing the data in five different intensities, where 1 is the lowest level for light intensity and 5 is the highest light intensity. The data is converted into a heat map for easier visualization. The different light intensities are controlled by using the projector.

The dark blue region in Figure 4 indicates that it is either over-exposed or underexposed and they are set to $0 \mathrm{~mm}$. At the light intensity 1 , the three-step phase-shifting method (Figure 4(a)) shows a better result compared to Jiang's method (current HDR method) and proposed HDR method, Figure 4(b) and Figure 4(c). This is because the current HDR method is developed for the shiny surface which may not be able to include a broad range of reflectivity (Feng et al., 2018). As the light intensity increases, the component class 4 with a white rectangle line surrounding it, as shown in Figure 4(a), starts to fail and become over-exposed when undergoing the regular 3PSP method, as shown in the indicator at light intensity 2 under 3PSP method. This is because the white rectangular line surrounding it has a high reflective property and caused it to be over-exposed. The pinhole of the circuit board from the three-step phase-shifting method is producing error as in Figure 4(d). The shiny surface of the sample cannot be seen when the reflectivity 


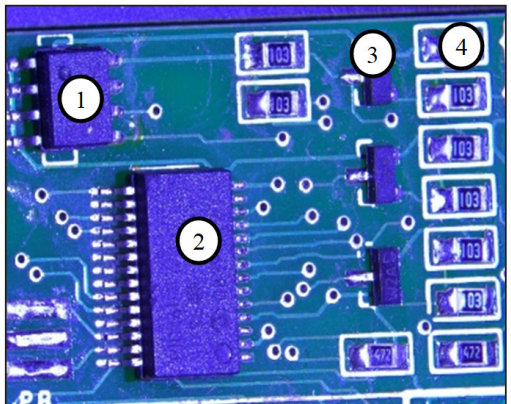

(a)

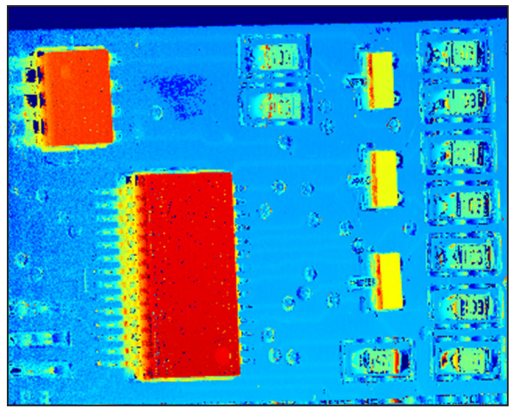

(c)

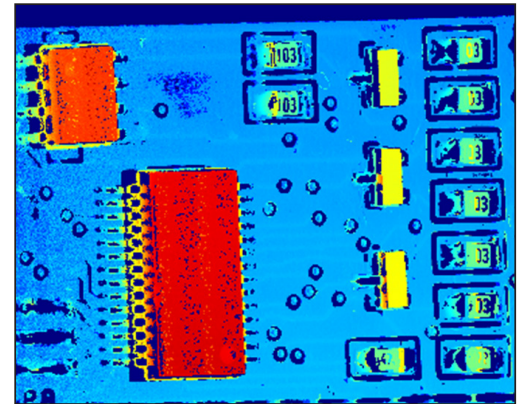

(b)

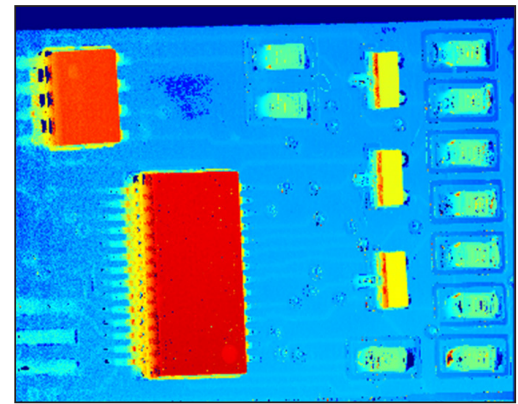

(d)

Figure 3. (a) Original image (b) Three-step phase-shifting (c) Current HDR method (d) Proposed HDR Method of the circuit board image

increases as in Figure 4(d). The proposed HDR method, shown in Figure 4(f), and current HDR method, shown in Figure 4(e) are clearly much better comparing with the regular three-step phase-shifting method at light intensity 2. On the other hand, the current HDR method shows improvement as the intensity increases but error starts to appear on the high reflective surface on component class 4 at light intensity 3 under current HDR method (Figure 4(h)), whereas, the proposed method is still able to give good results (Figure 4(i)).

At intensity 4, from the three-step phase-shifting (Figure 4(j)), the black IC is starting to become over-exposed and it is starting to produce error as shown in the indicator. The pinhole of the circuit board from the current method is also starting to get worse as the light intensity is increased (Figure 4(k)). However, the proposed method is still able to produce a satisfying result (Figure 4(1)). At intensity 5 of the three-step phase-shifting, the white part and the shiny part of the circuit board is over-exposed (Figure 4(m)). The result is as expected because the three-step phase-shifting algorithm cannot compute samples with surfaces that have high dynamic range of reflectivity. The current HDR method, however, shows better results, but the white part and the shiny part of the circuit board shows noise and some cannot be computed (Figure 4(n)). The proposed algorithm can compute the 3D data even at a high light intensity on the surface of the sample with a high dynamic range of reflectivity (Figure 4(o)). 


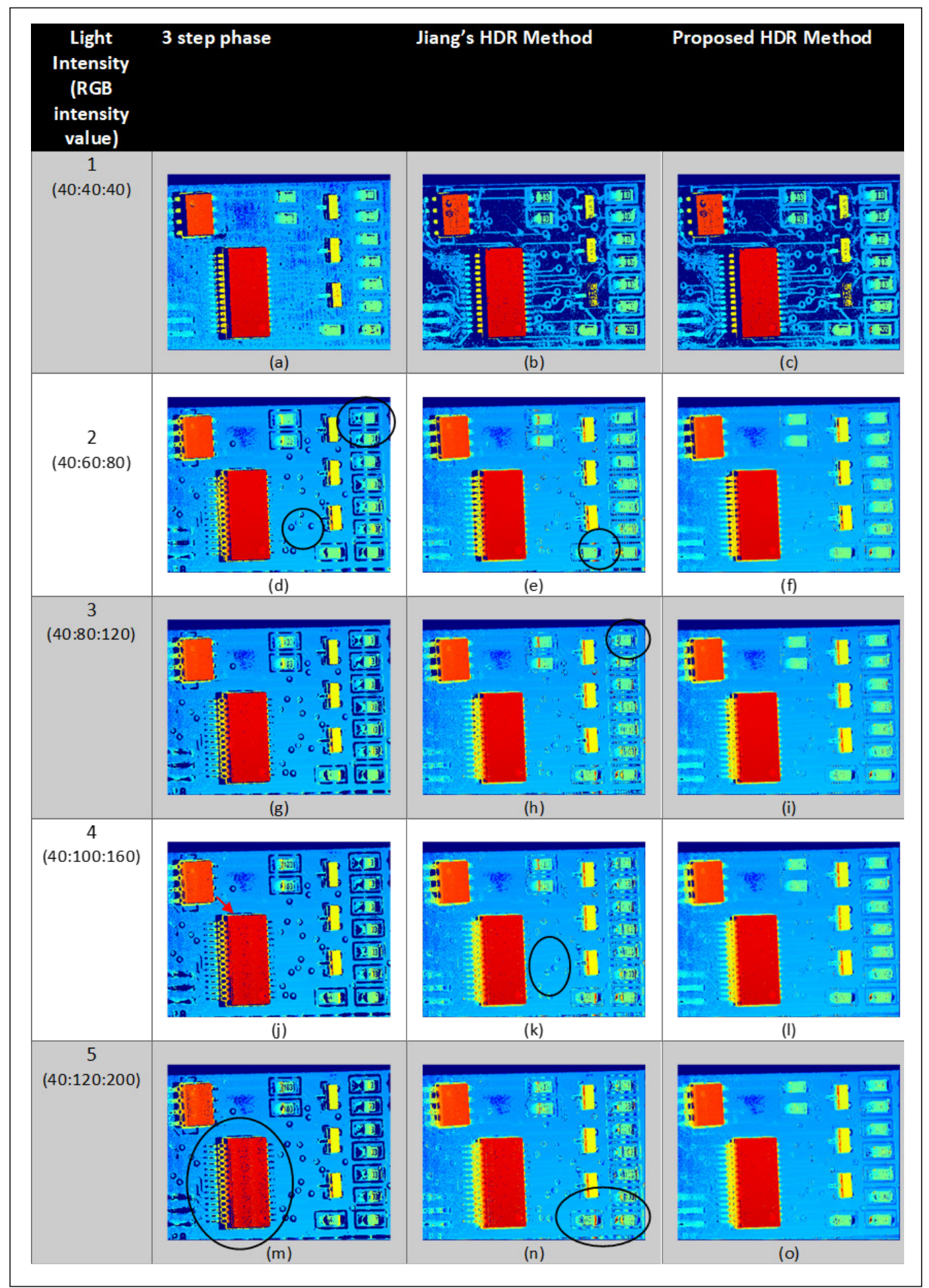

Figure 4. Comparison between the 3 step phase-shifting method, current HDR method and proposed HDR method at different light intensities 
Figure 6 displays the reconstruction of the solder ball grid from Figure 5. Solder ball grid shown in Figure 5 has both the highly reflective surface and low reflective surface. The result in Figure 6(a) indicates that the three-step phase shifting is not able to reconstruct the background of the image perfectly as it is brighter and more reflective. Not only that, but the tip of the solder balls is also not well reconstructed. Next, Jiang's HDR method in Figure 6(b) shows better result in the reconstruction of the solder ball tip, however, the background acquired is worse compared to the three-step phase-shifting method. The proposed method in Figure 6(c) shows a better reconstruction of the overall image compared to the two method. The background can be reconstructed even when it is highly exposed. Through the action of controlling the intensity of each RGB channel, we can reconstruct both the overexposed region and under-exposed region at a greater range which shows the robustness of the proposed HDR method.

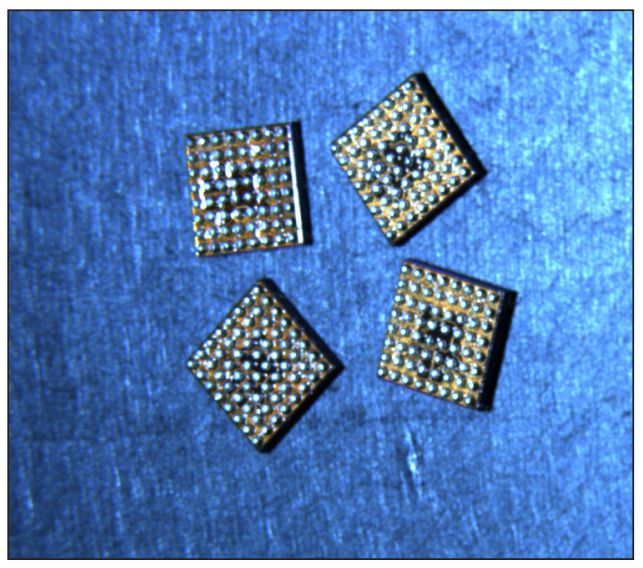

Figure 5. Solder ball grid

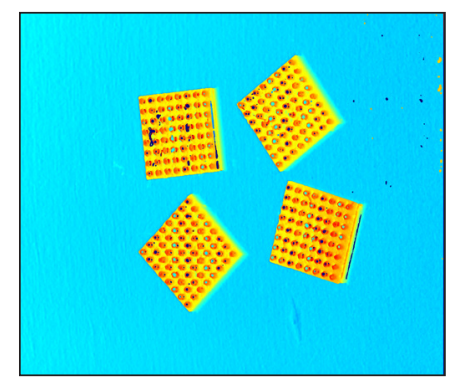

(a)

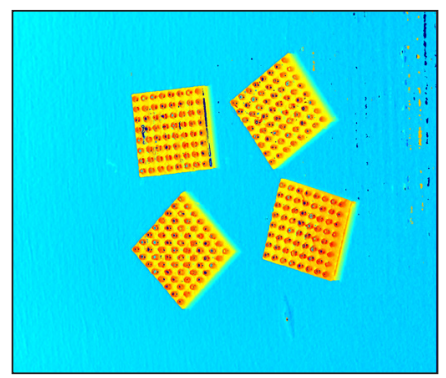

(b)

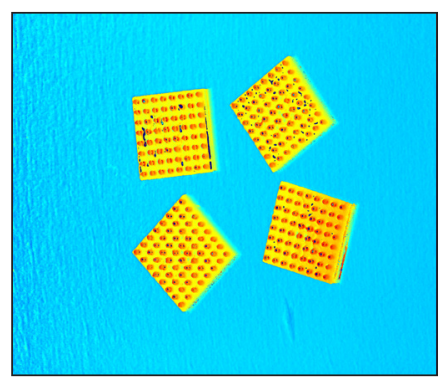

(c)

Figure 6. 3D reconstruction of solder ball grid (a) Three-step phase shifting (b) Jiang's HDR method (c) Proposed HDR method

\section{CONCLUSION}

This paper has shown an enhancement of the current HDR method. The proposed method can increase the dynamic range of the 3D shape measurement by controlling different channels of colour projected and isolating each channel.

To increase the dynamic range of the surface reflectivity, the RGB channels are used to control the intensity projected. Not only that, each RGB channels can compute the $3 \mathrm{D}$ data by itself. The experiment demonstrates that the 3D shape measurement can be successfully measured even at higher intensity while the current method had already overexposed. 
The current development of the HDR method that uses multiple camera exposures or multiple projector intensity requires many sets of images to be taken. This causes the process to be slow. Our proposed method can capture only one set of images and compute the HDR image. Consequently, our proposed method significantly reduces the $3 \mathrm{D}$ objects measurement times. However, our proposed method has the limitations of being unable to compute the $3 \mathrm{D}$ data at low exposure and there is no quality checking of the fringe captured.

\section{ACKNOWLEDGEMENT}

The equipment was provided by Estek Automation Sdn Bhd. This study would not be made possible without the help from Estek Automation Sdn Bhd.

\section{REFERENCES}

Aboali, M., Manap, N. A., Darsono, A. M., \& Yusof, Z. M. (2017). Review on three dimensional (3-D) acquisition and range imaging techniques. International Journal of Applied Engineering Research, 12(10), 2409-2421.

Achar, S., Bartels, J. R., Whittaker, W. L., Kutulakos, K. N., \& Narasimhan, S. G. (2017). Epipolar time-offlight imaging. ACM Transactions on Graphics, 36(4), 1-8. https://doi.org/10.1145/3072959.3073686

Babaie, G., Abolbashari, M., \& Farahi, F. (2015). Dynamics range enhancement in digital fringe projection technique. Precision Engineering, 39, 243-251. https://doi.org/10.1016/j.precisioneng.2014.06.007

Chen, C., Gao, N., Wang, X., \& Zhang, Z. (2018). Adaptive projection intensity adjustment for avoiding saturation in three-dimensional shape measurement. Optics Communications, 410(October 2017), 694701. https://doi.org/10.1016/j.optcom.2017.11.009

Faes, M., Abbeloos, W., Vogeler, F., Valkenaers, H., Coppens, K., Goedemé, T., \& Ferraris, E. (2016). Process Monitoring of Extrusion Based 3D Printing via Laser Scanning. Computer Vision and Pattern Recognition, 2016, 1-5. https://doi.org/10.13140/2.1.5175.0081

Feng, S., Zhang, L., Zuo, C., Tao, T., Chen, Q., \& Gu, G. (2018). High dynamic range 3-D measurements with fringe projection profilometry: A review. Measurement Science and Technology, 29(12), Article 122001. https://doi.org/10.1016/j.vetmic.2007.04.014

Huang, S. H., \& Pan, Y. C. (2015). Automated visual inspection in the semiconductor industry: A survey. Computers in Industry, 66, 1-10. https://doi.org/10.1016/j.compind.2014.10.006

Jiang, C., Bell, T., \& Zhang, S. (2016). High dynamic range real-time 3D shape measurement. Optics Express, 24(7), Article 7337. https://doi.org/10.1364/OE.24.007337

Liu, Y., Xi, J., Yu, Y., \& Chicharo, J. (2010). Phase error correction based on Inverse Function Shift Estimation in Phase Shifting Profilometry using a digital video projector. In Optical Metrology and Inspection for Industrial Applications (Vol. 7855, p. 78550W). International Society for Optics and Photonics. https:// doi.org/10.1117/12.870369 
Malamas, E. N., Petrakis, E. G., Zervakis, M., Petit, L., \& Legat, J. D. (2003). A survey on industrial vision systems,applications and tools. Image and Vision Computing, 21(2), 171-188. https://doi.org/10.1016/ S0262-8856(02)00152-X

Pavlicek, P., \& Mikeska, E. (2018). White-light interferometry without depth scan. In P. Zemánek (Ed.), 21st Czech-Polish-Slovak Optical Conference on Wave and Quantum Aspects of Contemporary Optics (Vol. 10976, p. 9). SPIE. https://doi.org/10.1117/12.2517877

Salahieh, B., Chen, Z., Rodriguez, J. J., \& Liang, R. (2014). Multi-polarization fringe projection imaging for high dynamic range objects. Optics Express, 22(8), Article 10064. https://doi.org/10.1364/OE.22.010064

Song, Z., Jiang, H., Lin, H., \& Tang, S. (2017). A high dynamic range structured light means for the 3D measurement of specular surface. Optics and Lasers in Engineering, 95(September 2016), 8-16. https:// doi.org/10.1016/j.optlaseng.2013.12.013

Waddington, C., \& Kofman, J. (2014). Modified sinusoidal fringe-pattern projection for variable illuminance in phase-shifting three-dimensional surface-shape metrology. Optical Engineering, 53(8), Article 084109. https://doi.org/10.1117/1.OE.53.8.084109

Wang, M., Du, G., Zhou, C., Zhang, C., Si, S., Li, H., Lei, Z., \& Li, Y. (2017). Enhanced high dynamic range 3D shape measurement based on generalized phase-shifting algorithm. Optics Communications, 385 , 43-53. https://doi.org/10.1016/j.optcom.2016.10.023

Wolff, L. B. (1989). Using polarization to separate reflection components. In Proceedings CVPR'89: IEEE Computer Society Conference on Computer Vision and Pattern Recognition (pp. 363-369). IEEE Conference Publication. https://doi.org/10.1109/CVPR.1989.37873

Zhang, S. (2016). High-speed 3D imaging with digital fringe projection techniques. CRC Press. https://doi. org/10.1017/CBO9781107415324.004

Zhang, S. (2018). High-speed 3D shape measurement with structured light methods: A review. Optics and Lasers in Engineering, 106(February), 119-131. https://doi.org/10.1016/j.optlaseng.2018.02.017

Zhang, S., \& Huang, P. S. (2006a). High-resolution, real-time three-dimensional shape measurement. Optical Engineering, 45(12), Article 123601. https://doi.org/10.1117/1.2402128,

Zhang, S., \& Huang, P. S. (2006b). Novel method for structured light system calibration. Optical Engineering, 45(8), Article 083601. https://doi.org/10.1117/1.2336196

Zhang, S., \& Yau, S. T. (2009). High dynamic range scanning technique. Optical Engineering, 48(3), Article 033604. https://doi.org/10.1117/1.3099720

Zuo, C., Feng, S., Huang, L., Tao, T., Yin, W., \& Chen, Q. (2018). Phase shifting algorithms for fringe projection profilometry: A review. Optics and Lasers in Engineering, 109(200), 23-59. https://doi.org/10.1016/j. optlaseng.2018.04.019 